\title{
PAIN INTERVENTION FOR TRIGEMINAL NEURALGIA
}

\author{
MA. Hadi ${ }^{1}$, A. Nor Zamzila ${ }^{2}$, AGM Rasydan ${ }^{1}$, N Suhaila ${ }^{1}$ and IM Nizamuddin ${ }^{1}$ \\ ${ }^{1}$ Departments of Anesthesiology and Critical Care, IIUM, Kuantan, Pahang, Malaysia, \\ ${ }^{2}$ Department of Basic Medical Science, IIUM, Kuantan, Pahang, Malaysia.
}

Presenter: Abdul Hadi, abhadi71@gmail.com

In the management of chronic pain, stepwise oral analgesics of graded strength are considered as first-line therapy. Minimally invasive interventional procedures remain an option for its treatment when pharmacological therapy fails to control the pain. We reported three classical cases of chronic trigeminal neuralgia that were managed with two types of pain intervention approaches after failing conservative management. In the first case, percutaneous pulse radiofrequency was directed directly to the trigeminal nerve while the other two patients were approached through Gasserian Ganglion. The first method used a traditional technique of pulse radiofrequency approaches. The nerve was identified using sensory stimulation and pulse radiofrequency was delivered once the nerve was confirmed. The second method was similar to the first one but with different approach. Under fluoroscopic control, a radiofrequency needle was introduced into the cheek's skin ipsilateral to the pain. The needle was directed towards the cranial base in front of the foramen ovale. The success of a trigeminal nerve block depends on proper identification of the anatomic landmarks and the nerve itself. The two different approaches of pain interventions offer more than $50 \%$ pain reduction. The trigeminal nerve approaches offer less risk as compared to Gasserian ganglion which is nearer to the brainstem and subarachnoid area. These procedures offer the ability to limit the sensory deficit to a preselected region of the face and to produce hypoalgesia or analgesia without anesthesia. 